\title{
Targeting the upstream transcriptional activator of PD-L1 as an alternative strategy in melanoma therapy
}

Bo Zhu ${ }^{1,13}$, Liming Tang ${ }^{2,3,13}$, Shuyang Chen ${ }^{1,13}$, Chengqian Yinn ${ }^{1,13}$, Shiguang Peng ${ }^{1,4}$, Xin $\mathrm{Li}^{1}$, Tongzheng $\mathrm{Liu}^{1}$, Wei Liu ${ }^{1,5}$, Changpeng Han ${ }^{1}$, Lukasz Stawski ${ }^{6}$, Zhi-Xiang $\mathrm{Xu}^{7}$, Guangbiao Zhou $^{8}$, Xiang Chen ${ }^{9}$, Xiumei Gao ${ }^{5}$, Colin R. Goding ${ }^{10}$, Nan Xu ${ }^{11^{*}}$, Rutao Cui ${ }^{*}$, Peng Cao ${ }^{2,12^{*}}$

1. Department of Pharmacology \& Experimental Therapeutics, Boston University School of Medicine, Boston, MA 02118, USA

2. Affiliated Hospital of Integrated Traditional Chinese and Western Medicine, Nanjing University of Chinese Medicine, Nanjing, Jiangsu, 210028, China

3. Affiliated Changzhou No. 2 People’s Hospital, Nanjing Medical University, Changzhou, Jiangsu, China

4. Department of Dermatology, Beijing Chaoyang Hospital, Capital Medical University, Beijing 100020, China

5. Tianjin State Key Laboratory of Modern Chinese Medicine, Tianjin University of Traditional Chinese Medicine, Tianjin, 300193, China

6. Arthritis Center/Section of Rheumatology, Boston University School of Medicine, Boston, MA 02118, USA

7. Division of Hematology \& Oncology, Department of Medicine, University of Alabama at Birmingham School of Medicine, Birmingham, AL 35233, USA

8. State Key Laboratory of Membrane Biology, Institute of Zoology, Chinese Academy of Sciences, 1 Beichen West Road, Chaoyang District, Beijing, 100101, China

9. Hunan key Laboratory of Skin Cancer and Psoriasis \& Department of Dermatology, Xiangya Hospital, Central South University, 87 Xiangya Rd, Changsha, Hunan, 410008, China

10. Ludwig Institute for Cancer Research, University of Oxford, Headington, Oxford, OX3 7DQ, UK

11. Dermatology department, Shanghai East Hospital, Tongji University, Shanghai, 200120, China

12. Collaborative Innovation Center for Cancer Medicine, Nanjing Medical University, Nanjing 211166, China

13. These authors contributed equally to this work

*To whom correspondence should be addressed:

Rutao Cui: rutaocui@bu.edu; Nan Xu: xnhrb@aliyun.com or Peng Cao: pcao79@yahoo.com

Running Title: Targeting PD-L1 activator in melanoma therapy 


\section{Abstract}

Programmed cell death ligand 1 (PD-L1) interacts with programmed cell death protein-1 (PD1) as an immune checkpoint. Reactivating the immune response by inhibiting PD-L1 using therapeutic antibodies provides substantial clinical benefits in many, though not all, melanoma patients. However, transcriptional suppression of PD-L1 expression as an alternative therapeutic anti-melanoma strategy has not been exploited. Here we provide biochemical evidence demonstrating that ultraviolet radiation (UVR) induction of PD-L1 in skin is directly controlled by nuclear factor E2-related transcription factor 2 (NRF2). Depletion of NRF2 significantly induces tumor infiltration by both $\mathrm{CD}^{+}$and $\mathrm{CD} 4^{+} \mathrm{T}$ cells to suppress melanoma progression, and combining NRF2 inhibition with anti-PD-1 treatment enhanced its anti-tumor function. Our studies identify a critical and targetable PD-L1 upstream regulator and provide an alternative strategy to inhibit the PD-1/PD-L1 signaling in melanoma treatment.

Keywords: PD-L1; melanoma; cancer immunotherapy; NRF2 


\section{Introduction}

The role of programmed death-1 (PD-1, CD279) as a "rheostat" of immunological regulation is attracting increasing attention (1). In response to binding its ligands including PD-L1, also named B7-H1 or CD274 and PD-L2 named with B7-DC or CD273, PD-1 represses the activation and function of $\mathrm{T}$ cells to downregulate the immune response (2). Usually, PD-1 expression is induced after T-cell activation, for example by ligation of T cell receptor (TCR) (3). PD-1/PD-L1 signaling plays inhibitory roles on cytokine production including TNF- $\alpha$, IFN- $\gamma$ and IL-2 (2). Studies found that loss of PD-1 contributes to mice resistance to viral infection, and inhibits tumor growth and metastasis through decrease of the antigen-recognition threshold and enhancement of $\mathrm{CD}^{+} \mathrm{T}$ cell cytotoxicity activity (4). Humanized antibodies against PD-1 or PD-L1, such as pembrolizumab (5), nivolumab (6), atezolizumab (7) and avelumab (8), have achieved promising clinical results in treatment of metastatic melanoma and other tumors by enhancing the immunity of tumor microenvironment and have been approved by the FDA $(9,10)$. In clinical trials of these PD-1/PD-L1 antibodies for advanced melanoma, response rates were $25 \%$ to $38 \%$ in patients and most responses were durable (11, 12). However, more and more studies indicate that acquired resistance is usually diagnosed after PD-1 antibody treatment $(10,11)$. To bypass the acquired resistance, different combination therapies are being tested for advanced melanoma, including pembrolizumab in combination with vemurafenib (BRAF inhibitor) and trametinib (MEK inhibitor) (13), and nivolumab in combination with ipilimumab (anti-CTLA-4 antibody) (14). However, alternative strategies of PD-1/PD-L1 inhibition therapy are also required to overcome acquired resistance, 
providing more options in new combination therapeutic strategies to improve clinical achievements for patients with melanoma.

Understanding the impact of driving genetic aberrations and signaling transduction in melanoma subsequently led to the identification of several targeted therapies that have proved effective in treating melanoma (15). One approach to improve PD-1/PD-L1 targeted therapies is to identify signaling pathways and transcription factors regulating PD-L1 expression. To date, a few upstream regulators have been identified, including p53, PTEN, AKT-mTOR, and NF$\kappa \mathrm{B}(16-18)$. As an oncogenic signaling mediator, AKT-mTOR pathway is shown as a significant therapeutic target in cancer treatment and mTOR inhibitor rapamycin combined with a PD-1 antibody showed promoted antitumor efficacy in a mouse model of lung cancer (18). However, it seems like that additional regulators on PD-L1 expression will provide valuable therapeutic targets in the drive to enhance immune checkpoint therapies.

NRF2, encoded by the NFE2L2 gene in human, is a transcription factor that regulates the expression of antioxidant proteins to protect cells against oxidative damage triggered by injury and inflammation (19). Interestingly, NRF2 activation promotes the development of de novo cancerous tumors (20) and several independent groups demonstrated that NRF2 functions as an anti-inflammatory regulator. For example, Nrf2 knockout mice show a tendency for agedependent autoimmune (21). Nrf2 loss or chemical blockade induces inflammation (22), and NRF2-targeted activators such as dimethyl fumarate have been successfully developed for clinical uses in patients with the autoimmune disease multiple sclerosis (23). All these studies 
indicated that NRF2-targeted inhibition has a strong rationale to be tested in melanoma treatment. In this work, we reveal that NRF2 regulates PD-L1 expression and NRF2-targeted therapy inhibits melanoma progression, which show potential to alternative strategy of PD1/PD-L1 antibody treatment in melanoma therapy. 


\section{Results}

\section{PD-L1 is dramatically up-regulated by UVB exposure}

Inhibition of the PD-1/PD-L1 checkpoint pathway by antibodies targeting PD-1 and PD-L1 was approved by FDA for treatment of metastatic melanoma, whereas activation of the pathway with recombinant PD-L1-Fc is effective in the treatment of the autoimmune disease psoriasis (24). Meanwhile, phototherapy with narrow-band ultraviolet-B (NB-UVB) is another effective immunosuppressive therapeutic strategy for patients with psoriasis (25). These observations raised the possibility that the PD-1/PD-L1 checkpoint pathway is involved in NBUVB treatment of psoriasis. To identify whether PD-1/PD-L1 is a UVB response factor in the NB-UVB treatment in patients with psoriasis, we examined PD-L1 protein expression in psoriatic skin after NB-UVB treatment. Interestingly, we found that the expression of PD-L1 in epidermis was strongly upregulated after UVB irradiation in vivo (Figure 1a).

This observation was further confirmed by flow cytometry of human primary keratinocytes (HPKs) and melanocytes (HPMs) after UVB irradiation in vitro, showing that PD-L1 protein expression was significantly upregulated after UVB exposure $\left(100 \mathrm{~J} / \mathrm{m}^{2}\right)$ in HPKs and HPMs (Figure 1b and c). UVB-induced CHK1 and p38 activation served as the positive controls. As a point of reference, a UVB dose of $100 \mathrm{~J} / \mathrm{m}^{2}$ is commonly used as a measure of sunlight and equate to one standard erythema dose (SED) with ambient exposure on a clear summer day in Switzerland being approximately 30-40 SED. An exposure dose of 4 SED is effective in psoriasis treatment and would be expected to produce moderate erythema on unacclimatised white skin but minimal or no erythema on previously exposed (tanned) skin (26). 
To test whether the upregulation of PD-L1 by UVB is at the transcriptional level, we extracted total RNA from UVB-treated HPKs and HPMs, and measured PD-L1 mRNA expression by RT-qPCR. As shown in Figure 1d and e, UVB exposure markedly induced expression of PDL1 mRNA up to 8-fold in HPKs and 5-fold in HPMs 6 hours after UVB exposure, which increased up to 15-fold in HPKs and 12-fold in HPMs at 24 hours after UVB exposure. UVB might affect some transcript stability to modulate the expression of some stress-inducible genes (27), such as p21, JUN, and RHOB (28-30). To test whether PD-L1 induction by UVB is due to altered mRNA stability, HPKs and HPMs cells were incubated with actinomycin D (10 nM) for one hour and then irradiated with UVB $\left(100 \mathrm{~J} / \mathrm{m}^{2}\right)$ or not. Cells were collected for total RNA extraction over time after UVB exposure and PD-L1 mRNA was assayed by RT-qPCR. The level of $\beta$-actin served as an internal loading control, since the quantity and stability of $\beta$ actin mRNA are not changed with UVB exposure (29). No significant differences in PD-L1 mRNA stability were observed between UVB-treated and non-treated cells (Supplementary

Figure 1a and b). These data suggest that PD-L1 is induced at the transcriptional level after UVB exposure.

\section{UVB-induced PD-L1 upregulation is dependent on NRF2}

To identify the potential transcription factor responsible for the activation of PD-L1 after UVB exposure, we utilized a web tool known as the Transcription Element Search System to detect potential transcription factors binding the promoter and intron regions of the PD-L1 gene. Besides the reported transcription factors IRF1(31), HIF-1(32), STAT1/3(31) and NF-אB(33), 
we also identified NRF2 binding site on PD-L1 gene. NRF2 is of particular interest since it is UVB-responsive and plays a key role in the cellular defense against oxidative stress and chronic inflammation in skin (19). Specifically, we found a potential NRF2-binding site (NBE) at 1932 to 1948 (CTTGAATGAACGGTCAC) (34) in the first intron of the PD-L1 gene that is conserved among human, mouse and rat (Figure 2a).

Consistent with NRF2 being UVB responsive, NRF2 protein expression was upregulated by UVB exposure in HPKs and HPMs (Figure 2b). To determine if there was any correlation between NRF2 and PD-L1 expression in melanoma, we examined melanoma tissue array slices of ME1003 from Biomax using immunohistochemistry. We found that NRF2 was detected in 27 of 52 primary melanomas (51.9\%), and 11 of 16 metastatic melanomas (68.8\%) and PD-L1 was detected in 28 of 52 primary melanomas (53.8\%), and 11 of 16 metastatic melanomas (68.8\%). Among all tested specimens, including benign tumors, NRF2 was detected in 18 (100\%) of the 18 specimens with high PD-L1 expression, but in only 23 (69.7\%) of the 33 specimens with low PD-L1 expression (Supplementary Table 1). The expression of PD-L1 is significantly correlated to NRF2 ( $r=0.7768, p<0.0001, \mathrm{n}=92$ ) (Figure 2c). To investigate the modulation of NRF2 on PD-L1, $N r f 2^{--}$and wild type mice were exposed to UVB with a dose of $500 \mathrm{~J} / \mathrm{m}^{2}$ on dorsal skin. The dorsal skin cells were isolated, and western blot assay were performed to detect the NRF2 expression (Figure 2d). As expected, UVB exposure significantly upregulated PD-L1 transcript to around 8 folds in dorsal skin cells of wild type mice, but UVB showed no effects on PD-L1 level in $\mathrm{Nrf2}^{-/-}$mice by RT-qPCR (Figure 2e). The cytometry assay was also performed to assay the PD-L1 expression on keratinocytes (K14 
positive) and melanocytes (Dct positive) of dorsal skin cells in both wild type and $\mathrm{Nrf2}^{-/-}$mice with UVB treatment. The results showed that UVB promoted PD-L1 expression on both keratinocytes and melanocytes of wild type, but not in $\mathrm{Nrf2}^{-/ /}$mice (Figure 2f). Therefore, all these results suggest that UVB-induced PD-L1 upregulation was dependent on NRF2.

\section{PD-L1 is a direct transcriptional target of NRF2}

To confirm that PD-L1 transcription is regulated by NRF2, we introduced NRF2 or empty vector into HPKs and HPMs (Figure 3a), and assessed PD-L1 mRNA and protein expression by RT-qPCR and flow cytometry, respectively. We found that PD-L1 expression was significantly induced in response to NRF2 overexpression at both the mRNA and protein levels (Figure $3 \mathbf{b}$ and $\mathbf{c}$ ). To verify if NRF2 is required in UVB-induced PD-L1 transcription, we generated HPKs and HPMs with stable NRF2 silencing by a specific shRNA knockdown (Figure 3d), and examined PD-L1 expression with $100 \mathrm{~J} / \mathrm{m}^{2} \mathrm{UVB}$ exposure. Cells with stable shNRF2 abrogated UVB-induced PD-L1 up-regulation at both the mRNA and protein levels (Figure 3e and f), indicating that NRF2 is required for PD-L1 up-regulation stimulated by UVB. These experiments suggest that PD-L1 is a NRF2-responsive gene.

To determine whether NRF2 protein binds to the regulatory region of the PD-L1 gene, we performed chromatin immunoprecipitation (ChIP) analysis of cross-linked chromatin isolated from UVB-irradiated (100 J/m $\left.\mathrm{m}^{2}\right)$ human primary melanocytes (HPMs) with immunoprecipitation of specific anti-NRF2 antibody and IgG control, followed by RT-qPCR assay for DNA enrichment using primers spanning the NRF2 binding site. The results revealed 
that NRF2 were enriched onto PD-L1 enhancer in HPMs cells with UVB treatment (Figure 3g). An intron control of PD-L1 without an NRF2 binding site, and the promoter of $\beta$-actin were used as the negative controls, and a promoter with an ARE (antioxidant response element) from the $\mathrm{NAD}(\mathrm{P}) \mathrm{H}$ quinone oxidoreductase 1 (NQO1) gene, a documented NRF2 target, served as a positive control (35). These data suggest that NRF2 directly recruit to modulate activation of the PD-L1 regulatory region. To further confirm the result, we generated luciferase reporter vectors in which luciferase is driven by the PD-L1 promoter alone, the promoter plus the wild-type NBE enhancer, or the promoter plus an enhancer with the NBE deleted. The luciferase assay results showed that the NBE-containing enhancer element increased PD-L1 promoter activity, which was abrogated by deleting the NRF2-binding site (Figure 3h). In addition, NRF2 introduction did not activate the PD-L1 reporter in the absence of the promoter region (Supplementary Figure 2a). NRF2-enhanced PD-L1 promoter activity is PD-L1 promoter sequence dependent. However, IFN- $\gamma$-stimulated PD-L1 transcriptional activation was repressed in cells with stable shNRF2 (Supplementary Figure 2b). These results suggest that NRF2 is required in IFN- $\gamma$-stimulated PD-L1 transcriptional activation.

\section{NRF2 modulates activation of anti-tumor $T$ cells}

Given facts that PD-L1 inhibition inhibits melanoma growth and NRF2 activates PD-L1 transcription, NRF2 should be a critical immune factor in melanoma. To identify whether NRF2-activated PD-L1 expression in melanoma cells suppresses lymphocyte cytotoxicity, B16 melanoma and antigen-specific TCR transgenic (Pmel-1) CD8 ${ }^{+}$T cells, which recognize gp100 melanoma antigen on B16 cells, were used to identify the role of NRF2 overexpression in 
inhibiting lymphocyte cytotoxicity. The targeted B16 melanoma cells were labeled with high concentration of CFSE and co-cultured with activated Pmel-1 T cells. Non-targeted LLC tumor cells labeled with low concentration of CFSE served as a control. We found that B16 tumor cells with NRF2 overexpression were significantly resistant to cytotoxicity of Pmel-1 T cells (Figure 4a). We also observed the secretion of IFN- $\gamma$ was repressed from Pmel-1 T cells incubated with B16 overexpressing NRF2 (Figure 4b). Some cytokine mRNAs were also detected by RT-qPCR in Pmel-1 T cells co-cultured with NRF2-overexpressing B16 cells. We found that the mRNA expression of IFN- $\gamma$, IL-2 and TNF $\alpha$ were repressed in Pmel-1 T cells after co-culturing with B16 cells with NRF2 overexpression (Figure 4c). Furthermore, we investigated whether NRF2 silencing in melanoma cells represses lymphocyte cytotoxicity. B16 melanoma cells with stable NRF2 silencing (shNRF2-B16) were co-cultured with gp100activated Pmel-1 T cells. Cytotoxicity activity was significantly enhanced in Pmel-1 T cells after incubating with B16 cells with stable shNRF2 (Figure 4d). Consistently, secretion of IFN- $\gamma$, IL-2 and TNF $\alpha$ cytokines were significantly upregulated in Pmel-1 T cells after incubating with B16 cells with stable shNRF2 (Figure 4e-f). To confirm anti-tumor cytotoxicity enhanced by NRF2 depletion depends on inhibition of PD-L1, we co-cultured Pmel-1 T cells with NRF2 depleted B16 cells with PD-L1 overexpression (Supplementary Figure 3a). We found that PD-L1 overexpression overcame the enhancement of Pmel-1 T cell cytotoxicity induced by co-culturing with NRF2 depleted B16 cells (Supplementary Figure 3b). Consistently, with PD-L1 overexpression NRF2 depleted failed to induce the cytokine mRNA expression of IFN- $\gamma$, IL-2 and TNF $\alpha$ in Pmel-1 T cells (Supplementary Figure 3c). All these data suggest that NRF2-regulated PD-L1 expression in melanoma cells modulates T 
cell activity. Thus, knockdown of NRF2 in melanoma cells is potentially to inhibit melanoma growth.

\section{NRF2 depletion cooperates with PD-1 blockade on melanoma treatment}

It is well known that PD-1 antibody treatment activates tumor-infiltrating $\mathrm{T}$ cells, which is effective in patients with melanoma (36). We therefore investigated whether PD-1 antibody in combination with NRF2 silencing augmented effector T-cell infiltration more efficiently than PD-1 antibody alone in melanoma. To address this question, an allograft melanoma mouse model was used with C57BL/6 mice ( $\mathrm{n}=5$ per group). Specifically, $2 \times 10^{4} \mathrm{~B} 16$ cells with stable NRF2 silencing or control shRNA were injected subcutaneously into 6-8 weeks old C57BL/6 mice. The mice were administered with intraperitoneal injection of $5 \mathrm{mg} / \mathrm{kg}$ control IgG or PD1 antibody from day 10 to day 15 . Tumor size was measured using calipers every three days and estimated from the formula: Tumor volume $\left(\mathrm{cm}^{3}\right)=\left(\right.$ length $\times$ width $\left.{ }^{2}\right) / 2$, and all mice were euthanized on day 21. As shown in Figure 5a-b, NRF2 depletion or PD-1 antibody treatment significantly inhibited melanoma tumor growth, and combination treatment showed synergetic anti-tumor function. To confirm the NRF2 depletion-induced PD-L1 decrease was efficient and consistent during allograft assay, we detect the PD-L1 expression from the allograft B16 cells at day 21. The flow cytometry results confirmed the decreased PD-L1 level by shNRF2 on B16 cells in allograft assay (Figure 5c). Tumor cell suspensions were prepared by digestion with collagenase of excised allograft tumors. After incubating with purified anti-CD16/32 Fc blocking antibodies, tumor-infiltrating lymphocytes (TILs) were stained with anti-CD45, antiCD3e, anti-CD8 and anti-CD4 mAbs, and then analyzed by flow cytometry. We found that 
combination therapy of NRF2 silencing and PD-1 antibody more significantly augmented tumor infiltration of $\mathrm{CD}^{+}\left(\mathrm{CD} 45^{+} \mathrm{CD}^{+} \mathrm{CD}^{+}\right) \mathrm{T}$ cells and $\mathrm{CD}^{+}\left(\mathrm{CD} 45^{+} \mathrm{CD}^{+} \mathrm{CD}^{+}\right) \mathrm{T}$ cells (Figure 3d and e) and repressed the fraction of TILs composed of Treg cells $\left(\mathrm{CD}^{+} \mathrm{CD}^{+} 5^{+} \mathrm{Foxp}^{+}\right)$than PD-1 antibody treatment alone (Figure 3f). To further evaluate the effects of NRF2 depletion on tumor-infiltrating $\mathrm{T}$ effector cell activation, we detected the granzyme $\mathrm{B}^{+}\left(\mathrm{GzmB}^{+}\right)$and IFN $\gamma^{+} \mathrm{CD}^{+} \mathrm{T}$ cells in TILs. We found that the percentage of both $\mathrm{GzmB}^{+} \mathrm{CD}^{+}$and $\mathrm{IFN} \gamma^{+} \mathrm{CD}^{+}$in total $\mathrm{CD}^{+}$TILs was increased after treatment with PD-1 antibody or NRF2 depletion alone (Figure $\mathbf{3 g}$ and $\mathbf{h}$ ). Interestingly, combination treatment of PD-1 antibody and NRF2 depletion significantly enhanced the percentage of $\mathrm{GzmB}^{+} \mathrm{CD}^{+}$and IFN $\gamma^{+} \mathrm{CD}^{+} \mathrm{T}$ cell than PD-1 antibody treatment or NRF2 depletion alone. These results suggest a synergistic benefit of PD-1 antibody and NRF2 depletion on cytokine production and cytotoxic granule secretion (Figure $\mathbf{3 g}$ and $\mathbf{h}$ ).

The tumor microenvironment is a complex structure, which includes myeloid cells represented by population of myeloid-derived suppressor cells (MDSCs) and tumor-associated macrophages (TAMs). MDSCs have immune regulatory properties and accumulate in tumors, blood, BM, spleen, and liver in melanoma patients and tumor-bearing mice (37). Here, the MDSCs' ratio was detected by labeling $\mathrm{CD} 45^{+} \mathrm{CD} 11 \mathrm{~b}^{+} \mathrm{Gr}-1^{+}$in xenograft tumors. Xenograft tumors developed by B16 melanoma cells with NRF2 silencing showed decreased MDSC frequency compared with negative controls. In addition, NRF2 depletion inhibited MDSC frequency synergistically with anti-PD-1 treatment in B16 allograft mouse models (Figure 5i). However, TIL quantification revealed that xenograft tumors developed by B16 melanoma cells 
with stable NRF2 silencing have similar portion of tumor-associated macrophage $\left(\mathrm{CD} 45^{+} \mathrm{CD} 11 \mathrm{~b}^{+} \mathrm{F} 4 / 80^{+}\right)$cell populations as in control IgG control group and in anti-PD-1 treatment mice (Figure 5j). However, NRF2 knockdown significantly increased the switch of an immunosuppressive M2-like $\left(\mathrm{CD} 11 \mathrm{~b}^{+} \mathrm{F} 4 / 80^{+} \mathrm{CD} 206^{+}\right)$phenotype to a more inflammatory

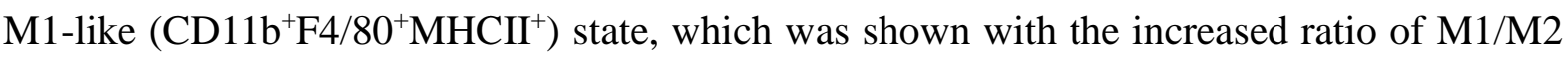
(Figure 5j). These results suggest that NRF2 depletion resulted in increase of activated T cells and decrease of suppressive myeloid cells, which enhanced the anti-tumor immunity in tumor environment to inhibit melanoma progression. 


\section{Discussion}

Targeting NRF2, an upstream transcriptional activator of PD-L1, is an alternative strategy in melanoma treatment. Initially, we found that PD-L1 is a UVB response gene (Figure 1), providing a molecular underpinning for UVB-induced immunosuppression. Phototherapy, the major therapeutic strategy for patients with psoriasis, induces apoptosis, immunosuppression and vitamin D production (38). Previous reports demonstrated that UVB-induced immunosuppression is triggered by UVB-induced DNA damage (39). Our studies reveal a new molecular mechanism for UVB-induced immunosuppression mediated by PD-L1/PD-1 signaling.

UVB-induced transcriptional activation of PD-L1 expression in a NRF2-dependent fashion (Figure 2 and 3). NRF2 is well-known to protect against chronic inflammatory through its ability to induce the expression of genes, including NADPH, quinone oxidoreductase (NQO1), several glutathione S-transferases (GST), and heme oxygenase 1 (HO-1) (40). The critical role of NRF2 in inflammation diseases has been confirmed in Nrf2 knockout mice, in which a severe autoimmune disease resembling systemic lupus erythematosus was diagnosed upon aging (41). Furthermore, the upregulation of expression of pro-inflammatory cytokines was detected in wounded skin of Nrf2 knockout mice (42). Here, we connect NRF2 with the critical PD-L1/PD-1 signaling pathway, providing a key mechanistic insight into the role of NRF2 protection in chronic inflammation.

The upstream activators of PD-L1 transcription have been extensively studied. Tp53-regulated 
miR-34 could binds to the PD-L1 transcript to represses its expression in non-small cell lung cancer cells (16). Cellular studies also demonstrated that activation of the AKT-mTOR pathway and inhibition of PTEN control PD-L1 expression (17). The IFN- $\gamma$-induced PD-L1 was modulated through activation of NF-אB (33), and STAT1/STAT2/STAT3-IRF1 axis to activate PD-L1 promoter (31). HIF-1 $\alpha$ is another transcription factor binding to HRE on PD-L1 promoter to upregulate PD-L1 expression response to hypoxia stress (32). However, the identification of any direct upstream transcription activators remains to be investigated. Here, we found that PD-L1 is directly regulated by the NRF2, which is induced with UVB treatment (Figure 3), and that NRF2-targeted therapy is a novel alternative strategy of PD-1/PD-L1 antibody treatment in melanoma (Figure 5).

PD-1 blockade was a breakthrough in melanoma immunotherapy. Humanized PD-1/PD-L1 antibodies, including pembrolizumab, nivolumab, BMS-936559 and avelumab $(10,12)$ have been developed with high response rates and efficacy for advanced melanoma, which are approved by FDA. Recently, different combination therapies have been tested for melanoma, including pembrolizumab in combination with vemurafenib or trametinib (13) to further improve the treatment achievement and overcome acquired resistance. Here, our studies indicate that targeted inhibition of NRF2 is an alternative strategy of PD-1/PD-L1 inhibition to activate infiltration T cells and subsequently inhibit melanoma growth (Figure 5). Previous histological, genetic and clinical observations have provided strong evidence that NRF2 inhibition is a potential therapeutic strategy in melanoma treatment $(20,43)$. Specifically, NRF2 expression was shown to be associated with malignance and less sensitivity to targeted 
therapy drugs in melanoma (44). Furthermore, genetically engineered mouse studies and clinical observation found that NRF2 activation promoted the development of cancerous tumors (20). We demonstrated that NRF2-targeted inhibition suppresses PD-L1 to enhance tumor-infiltrating $\mathrm{T}$ cell activation to inhibit melanoma growth and provided a new strategy in melanoma immunotherapy. However, NRF2-targeted inhibition only delayed tumor progression and death with combinatorial treatment, which are consistent with the results of clinical trials of PD-1 antibody treatment in melanoma $(6,45,46)$. Although around $30 \%$ melanoma patients showed response to anti-PD-1 treatment, tumor relapses within two years were observed in a significant proportion of those responding patients $(6,45,46)$. 


\section{Materials and methods}

\section{Cell lines and culture}

HPK and HPM were isolated as described previously (47). In summary, human primary keratinocyte medium with human keratinocyte growth supplement (HKGS, Life Technologies) were used for culture of HPKs, with Medium 254 with human melanocyte growth supplement (HMGS, Life Technologies) for HPMs. B16-BL6 cells were maintained in DMEM with 10\% FBS (ThermoFisher Scientific). All cell culture medium was in the presence of penicillin (100 $\mathrm{U} / \mathrm{mL})$ and streptomycin $(100 \mu \mathrm{g} / \mathrm{mL})$. For knockdown of NRF2, human shNRF2 (RHS4533EG4780) and mouse shNRF2 (RMM4534-EG18024) were purchased from Open Biosystems, GE Dharmacon, and packaged with psPAX2 (Addgene, \#12260) and pMD2.G (Addgene, \#12259) in HEK293T using Lipofectamine 3000 (Invitrogen). The efficient shNRF2 used are: human ATTTCACATCACAGTAGGAGC, mouse ATTAAGACACTGTAATTCGGG. Lentiviruses were collected after 48 hours transfection, and then were used to infect target cells for 24 hours in the presence of polybrene $(8 \mu \mathrm{g} / \mathrm{mL})$. Puromycin $(2 \mu \mathrm{g} / \mathrm{mL})$ was used for selection of stable cell lines. The NRF2 cDNA was amplified from pCDNA3-Myc3-Nrf2 (\#21555, Addgene) and subcloned into pQCXIP (Clonetech) vector. NRF2 constructs, pCMVVSV-G (\#8454, Addgene) and pUMVC (\#8449, Addgene) plasmids were transfected into HEK293T cells using Lipofectamine 3000. Retroviruses were harvested 48 hours posttransfection for infection target cells. Mouse PD-L1 (\#MG50010-CY, Sino Biological) was transfected into shNRF2 B16-BL6 stable cells for 2 days, before adding hygromycin (200 $\mu \mathrm{g} / \mathrm{mL}$ ) for stable cell line selection.

\section{Animal study}


All animal studies were performed in accordance with the Guide for the Care and Use of Laboratory Animals of the National Institutes of Health. The protocol was reviewed and approved by the Animal Science Center (ASC) of Boston University. Female C57BL/6J, Nrf2^- and Pmel-1 mice were purchased from the Jackson Laboratory and housed at the Animal Science Center (ASC) of Boston University School of Medicine. Allograft assay was performed with a total number of $2 \times 10^{4}$ cells with subcutaneous (s.c.) injection into C57BL/6J mice. Antibodies (5 mg/kg) of anti-PD-1 (RMP1-14, Bio X cell) and IgG control were s.c. injected into mice at day 10 after tumor cell inoculation. Tumor volumes were established every three days $\left(0.5^{*}\right.$ length $\times$ width $\left.^{2}\right)$. UVB treatment on mice were carried out in the Stratalinker UV chamber (Stratagene, Cedar Creek, TX, USA) with UVB bulbs (UVP LLC, Upland, CA, USA) with $500 \mathrm{~J} / \mathrm{m}^{2} \mathrm{UVB}$ on mouse dorsal skin.

\section{Mouse TIL isolation}

Dissected tumors were minced into small fragments for digestion with HBSS (Life Technologies) in presence of collagenase $(1 \mathrm{mg} / \mathrm{mL})$, hyaluronidase $(2.5 \mathrm{U} / \mathrm{mL})$ and DNAse I (0.1 mg/mL) (Sigma-Aldrich) for 2 hours at room temperature. Then cell suspends were go through a $70 \mu \mathrm{m}$ cell strainer to get the single-cell suspend.

\section{Flow cytometry}

HPK and HPM single-cell suspension were fixed with 2\% paraformaldehyde for 20 minutes, before blocked with human Fc Receptor Binding Inhibitor Purified (14-9161-71, eBioscience) for 30 minutes on ice. The cells were suspend in staining buffer (00-4222-26, eBioscience), 
and incubated with primary antibody PD-L1 PE (1:100, 12-5983-41, eBioscience) for 1 hour on ice. UVB-treated and control dorsal skins were isolated and incubated in PBS with EDTA $(5 \mathrm{mM})$ at $37^{\circ} \mathrm{C}$ for 2 hours to remove subcutis. The epithelial sheet were digested with collagenase $\mathrm{P}\left(1 \mathrm{mg} \mathrm{ml}^{-1}\right)$ and dispase $\left(1 \mathrm{mg} \mathrm{ml}^{-1}\right)$ at $37^{\circ} \mathrm{C}$ for half hour, and suspend to prepare the sing-cell suspend by through $70 \mu \mathrm{m}$ cell strainer. The mouse keratinocytes was labeled with anti-cytokeratin 14 (ab181595), and mouse melanocytes were labeled with anti-Dct (ab74073). For TILs of $\mathrm{CD}^{+}$and $\mathrm{CD} 4^{+} \mathrm{T}$ cells staining, single-cell suspension from tumor were stained as the above procedure, and identified with staining by antibodies including: anti-CD45 APC (104, BD pharmingen), anti-CD3e PE (145-2C11, BD pharmingen), anti-CD4 FITC (RM4-5, BD pharmingen) and anti-CD8 FITC (53-6.7, BD pharmingen). The regulatory T cells in TILs were stained with the Mouse Regulatory T Cell Staining kit\#1 (88-8111, ThermoFisher Scienctific), with antibodies of anti-mouse CD4 FITC (RM4-5), anti-mouse CD25 APC (PC61.5) anti-mouse Foxp3 PE (FJK-16s). Cell Activation Cocktail (with Brefeldin A) (Biolegend) was added to cell culture for 6 hours to detect the cytokine production in effector $\mathrm{CD}^{+} \mathrm{T}$ cells, and labeled with anti-CD8 FITC (53-6.7, BD pharmingen), anti-mouse granzyme B PE-Cy7 (NGZB, eBioscience) and anti-mouse IFN $\gamma$ PE (XMG1.2, eBioscience). For labeling MDSC, anti-CD11b PE-Cy7 (clone M1/70) and anti-Gr-1 FITC (RB6-8C5) were used. Antibodies of anti-F4/80 (BM8) FITC, anti-CD11b PE-Cy7 (clone M1/70), anti-CD206 PE (C068C2) and anti-I-A/I-E (MHCII) Pacific Blue (M5/114.15.2) were used for labeling tumorassociated macrophage. LSRII (BD Biosciences) was used to assay the samples, and data analysis was performed with FlowJo software. 


\section{Immunohistochemistry staining}

Fixed psoriasis patient skins samples were cut into $5 \mu \mathrm{m}$-thick sections. The sections were deparaffinized and rehydrated, and then were boiled water in $10 \mathrm{mM}$ sodium citrate buffer $(\mathrm{pH}$ 6.0) for 20 minutes for antigen retrieval. Tris Buffered Saline (TBS) with 0.1\% Tween-20 and 5\% normal goat serum were used for block. Primary antibodies of anti-PD-L1 (1:200) (13684, Cell Signaling Technology) and anti-NRF2 (1:200) (ab31163, Abcam) were incubated at $4{ }^{\circ} \mathrm{C}$ for overnight. After incubated with the corresponding secondary antibodies, slides were prepared with Pierce ${ }^{\mathrm{TM}}$ DAB Substrate Kit (ThermoFisher Scientific). Counterstaining was performed with hematoxylin.

\section{Melanoma tissue array}

The melanoma tissue arrays, ME1003, were purchased from Biomax, containing 56 cases of malignant melanoma, 20 cases of metastatic malignant melanoma and 24 benign tumor. The IHC staining of array was performed with antibodies of anti-PD-L1 (1:200) (13684, Cell Signaling Technology) and anti-NRF2 (1:200) (ab31163, Abcam). The association between NRF2 expression and PD-L1 expression was represented with Pearson correlation coefficient. The $p<0.05$ was considered as statistically significant.

\section{Quantitative RT-PCR analysis}

Quantitative RT-PCR (RT-qPCR) was performed as previously described (48). Specifically, RNA was prepared with Qiagen RNeasy mini kit for reverse transcription reaction using HighCapacity cDNA Reverse Transcription Kit (Applied Biosystems). The cDNA was used for 
qPCR with SYBR green PCR master mix (Applied Biosystems). The expression level of PDL1 was normalized to $\beta$-actin. All RT-qPCR assay were repeated for three time. The primers used in experiment include human PD-L1 forward: 5'-GCTGCACTAATTGTCTATTGGGA3', reverse: 5'-AATTCGCTTGTAGTCGGCACC-3'; human $\beta$-actin forward: 5'ATTGGCAATGAGCGGTTCC-3’, reverse: 5’-GGTAGTTTCGTGGATGCCACA-3’.

\section{Immunoblotting}

Cells lysis were prepared with RIPA buffer in the presence of protease inhibitor cocktail (ThermoFisher Scientific). Protein concentrations were assayed with Bradford Protein Assay Kit I (Bio-Rad). The same amount of protein samples were separated by SDS-PAGE gel, and were transferred onto a PVDF membrane. Membranes were blot with primary antibodies, which include anti-NRF2 (D1Z9C, Cell Signaling Technology), anti-p38 (D13E1, Cell Signaling Technology), anti-Phospho-p38 (Thr180/Tyr182) (D3F9, Cell Signaling Technology), anti-CHK1 (ab40866, Abcam), anti-Phospho-CHK1 (Ser345) (133D3, Cell Signaling Technology) and anti- $\beta$-actin (A5441, Sigma-Aldrich), and corresponding secondary antibodies for visualization.

\section{Chromatin immunoprecipitation (ChIP) assays}

The ChIP assay were performed as described previously (48). Specifically, cells $\left(1 \times 10^{7}\right.$ for each IP) were cross-linked with $1 \%$ formaldehyde for 10 minutes, and then stopped by adding glycine (0.125M). Cells pellets were suspend in ChIP lysis buffer, and cross-linked lysate was sonicated for DNA fragments. Immunoprecipitation was performed with antibodies anti-NRF2 
(D1Z9C, Cell Signaling Technology) and anti-rabbit IgG control. The chromatin complex was pulled down with protein A/G agarose beads (Invitrogen) and reversed the cross-lines at $65^{\circ} \mathrm{C}$ overnight. Eluted DNA was assayed with qPCR. Potential NRF2 binding sites were analyzed by regulatory VISTA (rVISTA) online tool (http://genome.lbl.gov/vista). All RT-qPCR results were calculated by comparative Ct method as described previously (48). Primers include NQO1 forward: 5'-TCCAAATCCGCAGTCACAGT-3', reverse: 5'TTGGCACGAAATGGAGC-3'; $\beta$-actin forward: 5'-AACTCTCCСТCCTCСTCTTCCTC-3', reverse: 5'-GAGCCATAAAAGGCAACTTTCGG-3'; Chr19 forward: 5'TGGGAAAACTCTCCAGGAC-3'， reverse: 5'-CTTTGGTTGCCTGTGCTT-3'; PD-L1 Enhancer forward: 5'-ATTACAGGCATGAGCCACTGCT-3', reverse: 5’TGACCATATGTGAATTCCCATATCCGA-3’; PD-L1 non-binding element forward: 5’CACCCAGGGAGCATCTGGT-3’, reverse: 5’-TCAGCACTGGCACTGTTGAC-3’.

\section{Procedures of NB-UVB phototherapy}

Psoriasis patient biopsy was treated with NB-UVB irradiation $\left(300 \mathrm{~J} / \mathrm{m}^{2}\right)$, and sham irradiation was performed as the control. After 6 hours post-irradiation IHC staining was performed with anti-PD-L1 (1:200) (13684, Cell Signaling Technology). Written informed consents were obtained from the participants. The study was approved by the Beijing Chaoyang Hospital Scientific and Ethics Committee and was conducted according to the principles of the Declaration of Helsinki.

\section{In vitro cell culture experiments}


Splenocytes were prepared from Pmel-1 mice, and activated by treated with gp100 peptide (1 $\mu \mathrm{M})$ for 24 hours. The activated T cells were cultured and rested with IL-2 (30 U/ml) for 4 days, and then the Pmel-1 cells were co-cultured with indicated B16 melanoma cells for 48 hours for IFN- $\gamma$ ELISA assay. The Pmel-1 cells were collected for detecting IFN- $\gamma$, IL-2 and TNF $\alpha$ with RT-qPCR. Pmel-1 T cell-targeted B16 melanoma cells and non-targeted control LLC cells were labeled with high concentration $(1 \mu \mathrm{M})$ and low concentration $(0.1 \mu \mathrm{M})$ of CFSE (eBioscience), and then co-cultured with activated Pmel-1 cells with the ration of 1:10 for 5 hours. The cells were then prepared for flow cytometry assay. The cytotoxicity was calculated as percentages of cytotoxicity with the formula: 100-

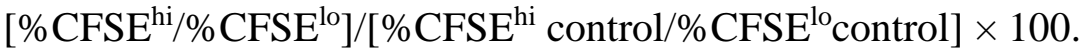

\section{Statistical analyses}

Results were calculated with the mean \pm standard deviation (SD) with three independent experiments. Two-tailed unpaired Student's $t$-test or analysis of variance was used to determine the statistical significance. $P$ values $<0.05$ were considered as significance $\left({ }^{*} p<0.05,{ }^{* *} p<0.01\right.$, and $* * * p<0.001)$. 


\section{Conflict of Interest Statement}

The authors declare no conflict of interest.

\section{Acknowledgments}

We thank Drs. Wenshu Wu and Rui He for carefully reading and suggestions. This work was supported by the National Institutes of Health (RC: R01CA137098, R01CA193913 and R01CA196896), Melanoma Research Foundation Establish Investigator Award (RC), Hong Kong, Macao Young Scientists of the National Natural Science Foundation of China (Grant No.81428025 for RC) and National Natural Science Foundation of China (81630106), and the Priority Academic Program Development of Jiangsu Higher Education Institutions (Integration of Chinese and Western Medicine) grant (PC). National Natural Science Foundation of China (31771619) for HH. The National Natural Science Foundation of China (Grant No. 81673977 for 2016 for XM). RC is an American Cancer Society Research Scholar.

\section{Author Contributions}

B.Z., L.T., S.C. and C.Y. performed most of the experiments with assistance from S.P., X.L., T.L., W.L., C.H., L.S., Z.X., G.Z., X.C. and X.G.. P.C., R.C. and N.X. designed and supervised the experiments. R.C., N.X. and P.C. wrote the manuscript with assistance from C.G.. 


\section{References}

1. Ishida Y, Agata Y, Shibahara K, Honjo T. Induced expression of PD-1, a novel member of the immunoglobulin gene superfamily, upon programmed cell death. EMBO J. 1992;11(11):3887-95.

2. Keir ME, Butte MJ, Freeman GJ, Sharpe AH. PD-1 and its ligands in tolerance and immunity. Annu Rev Immunol. 2008;26:677-704.

3. Nishimura H, Agata $Y$, Kawasaki A, Sato M, Imamura S, Minato N, et al. Developmentally regulated expression of the PD-1 protein on the surface of double-negative (CD4-CD8-) thymocytes. Int Immunol. 1996;8(5):773-80.

4. Fourcade J, Sun Z, Benallaoua M, Guillaume P, Luescher IF, Sander C, et al. Upregulation of Tim-3 and PD-1 expression is associated with tumor antigen-specific CD8+ T cell dysfunction in melanoma patients. J Exp Med. 2010;207(10):2175-86.

5. Gogas H, Abali H, Ascierto PA, Demidov L, Pehamberger H, Robert C, et al. Who benefits most from adjuvant interferon treatment for melanoma? Am J Ther. 2015;22(1):54-60.

6. Robert C, Long GV, Brady B, Dutriaux C, Maio M, Mortier L, et al. Nivolumab in previously untreated melanoma without BRAF mutation. N Engl J Med. 2015;372(4):320-30.

7. Romero D. Immunotherapy: Atezolizumab becomes POPLAR. Nat Rev Clin Oncol. 2016;13(5):266.

8. Sidaway P. Skin cancer: Avelumab effective against Merkel-cell carcinoma. Nat Rev Clin Oncol. 2016;13(11):652.

9. Ott PA, Hodi FS, Robert C. CTLA-4 and PD-1/PD-L1 blockade: new immunotherapeutic modalities with durable clinical benefit in melanoma patients. Clin Cancer Res. 2013;19(19):5300-9.

10. Topalian SL, Hodi FS, Brahmer JR, Gettinger SN, Smith DC, McDermott DF, et al. Safety, activity, and immune correlates of anti-PD-1 antibody in cancer. N Engl J Med. 2012;366(26):2443-54.

11. Ribas A, Hamid O, Daud A, Hodi FS, Wolchok JD, Kefford R, et al. Association of Pembrolizumab With Tumor Response and Survival Among Patients With Advanced Melanoma. JAMA. 2016;315(15):1600-9.

12. Hamid O, Robert C, Daud A, Hodi FS, Hwu WJ, Kefford R, et al. Safety and tumor responses with lambrolizumab (anti-PD-1) in melanoma. N Engl J Med. 2013;369(2):134-44.

13. Schadendorf D, Fisher DE, Garbe C, Gershenwald JE, Grob JJ, Halpern A, et al. Melanoma. Nat Rev Dis Primers. 2015;1:15003.

14. Larkin J, Chiarion-Sileni V, Gonzalez R, Grob JJ, Cowey CL, Lao CD, et al. Combined Nivolumab and Ipilimumab or Monotherapy in Untreated Melanoma. N Engl J Med. 2015;373(1):23-34.

15. Tsao H, Chin L, Garraway LA, Fisher DE. Melanoma: from mutations to medicine. Genes Dev. 2012;26(11):1131-55.

16. Cortez MA, Ivan C, Valdecanas D, Wang X, Peltier HJ, Ye Y, et al. PDL1 Regulation by p53 via miR-34. J Natl Cancer Inst. 2016;108(1).

17. Parsa AT, Waldron JS, Panner A, Crane CA, Parney IF, Barry JJ, et al. Loss of tumor suppressor PTEN function increases B7-H1 expression and immunoresistance in glioma. Nat Med. 2007;13(1):84-8.

18. Lastwika KJ, Wilson W, 3rd, Li QK, Norris J, Xu H, Ghazarian SR, et al. Control of PD-L1 Expression by Oncogenic Activation of the AKT-mTOR Pathway in Non-Small Cell Lung Cancer. Cancer Res. 2016;76(2):227-38.

19. Gorrini C, Harris IS, Mak TW. Modulation of oxidative stress as an anticancer strategy. Nature reviews Drug discovery. 2013;12(12):931-47.

20. DeNicola GM, Karreth FA, Humpton TJ, Gopinathan A, Wei C, Frese K, et al. Oncogene-induced Nrf2 transcription promotes ROS detoxification and tumorigenesis. Nature. 2011;475(7354):106-9.

21. Yoh K, Itoh K, Enomoto A, Hirayama A, Yamaguchi N, Kobayashi M, et al. Nrf2-deficient female mice develop lupus-like autoimmune nephritis. Kidney Int. 2001;60(4):1343-53. 
22. Rangasamy T, Cho CY, Thimmulappa RK, Zhen L, Srisuma SS, Kensler TW, et al. Genetic ablation of Nrf2 enhances susceptibility to cigarette smoke-induced emphysema in mice. J Clin Invest. 2004;114(9):1248-59.

23. Hybertson BM, Gao B, Bose SK, McCord JM. Oxidative stress in health and disease: the therapeutic potential of Nrf2 activation. Mol Aspects Med. 2011;32(4-6):234-46.

24. Kim JH, Choi YJ, Lee BH, Song MY, Ban CY, Kim J, et al. Programmed cell death ligand 1 alleviates psoriatic inflammation by suppressing IL-17A production from programmed cell death 1-high T cells. J Allergy Clin Immunol. 2016;137(5):1466-76 e3.

25. Menter A, Korman NJ, Elmets CA, Feldman SR, Gelfand JM, Gordon KB, et al. Guidelines of care for the management of psoriasis and psoriatic arthritis: Section 5. Guidelines of care for the treatment of psoriasis with phototherapy and photochemotherapy. J Am Acad Dermatol. 2010;62(1):114-35.

26. Heckman CJ, Chandler R, Kloss JD, Benson A, Rooney D, Munshi T, et al. Minimal Erythema Dose (MED) testing. J Vis Exp. 2013(75):e50175.

27. Sachs AB. Messenger RNA degradation in eukaryotes. Cell. 1993;74(3):413-21.

28. Blattner C, Kannouche P, Litfin M, Bender K, Rahmsdorf HJ, Angulo JF, et al. UV-Induced stabilization of cfos and other short-lived mRNAs. Mol Cell Biol. 2000;20(10):3616-25.

29. Wang W, Furneaux $\mathrm{H}$, Cheng $\mathrm{H}$, Caldwell MC, Hutter D, Liu Y, et al. HuR regulates p21 mRNA stabilization by UV light. Mol Cell Biol. 2000;20(3):760-9.

30. Westmark CJ, Bartleson VB, Malter JS. RhoB mRNA is stabilized by HuR after UV light. Oncogene. 2005;24(3):502-11.

31. Garcia-Diaz A, Shin DS, Moreno BH, Saco J, Escuin-Ordinas H, Rodriguez GA, et al. Interferon Receptor Signaling Pathways Regulating PD-L1 and PD-L2 Expression. Cell Rep. 2017;19(6):1189-201.

32. Noman MZ, Desantis G, Janji B, Hasmim M, Karray S, Dessen P, et al. PD-L1 is a novel direct target of HIF1alpha, and its blockade under hypoxia enhanced MDSC-mediated T cell activation. J Exp Med. 2014;211(5):78190.

33. Kondo A, Yamashita T, Tamura H, Zhao W, Tsuji T, Shimizu M, et al. Interferon-gamma and tumor necrosis factor-alpha induce an immunoinhibitory molecule, B7-H1, via nuclear factor-kappaB activation in blasts in myelodysplastic syndromes. Blood. 2010;116(7):1124-31.

34. Chorley BN, Campbell MR, Wang X, Karaca M, Sambandan D, Bangura F, et al. Identification of novel NRF2regulated genes by ChIP-Seq: influence on retinoid X receptor alpha. Nucleic Acids Res. 2012;40(15):7416-29.

35. Hybertson BM, Gao B. Role of the Nrf2 signaling system in health and disease. Clin Genet. 2014;86(5):44752.

36. Sato E, Olson SH, Ahn J, Bundy B, Nishikawa H, Qian F, et al. Intraepithelial CD8+ tumor-infiltrating lymphocytes and a high $\mathrm{CD} 8+$ /regulatory $\mathrm{T}$ cell ratio are associated with favorable prognosis in ovarian cancer. Proc Natl Acad Sci U S A. 2005;102(51):18538-43.

37. Umansky V, Sevko A, Gebhardt C, Utikal J. Myeloid-derived suppressor cells in malignant melanoma. J Dtsch Dermatol Ges. 2014;12(11):1021-7.

38. Clydesdale GJ, Dandie GW, Muller HK. Ultraviolet light induced injury: immunological and inflammatory effects. Immunol Cell Biol. 2001;79(6):547-68.

39. Hart PH, Gorman S, Finlay-Jones JJ. Modulation of the immune system by UV radiation: more than just the effects of vitamin D? Nat Rev Immunol. 2011;11(9):584-96.

40. Nguyen T, Yang CS, Pickett CB. The pathways and molecular mechanisms regulating Nrf2 activation in response to chemical stress. Free Radic Biol Med. 2004;37(4):433-41.

41. Li J, Stein TD, Johnson JA. Genetic dissection of systemic autoimmune disease in Nrf2-deficient mice. Physiol Genomics. 2004;18(3):261-72. 
42. Beyer TA, Auf dem Keller U, Braun S, Schafer M, Werner S. Roles and mechanisms of action of the Nrf2 transcription factor in skin morphogenesis, wound repair and skin cancer. Cell Death Differ. 2007;14(7):1250-4.

43. Ren D, Villeneuve NF, Jiang T, Wu T, Lau A, Toppin HA, et al. Brusatol enhances the efficacy of chemotherapy by inhibiting the Nrf2-mediated defense mechanism. Proc Natl Acad Sci U S A. 2011;108(4):1433-8.

44. Hintsala HR, Jokinen E, Haapasaari KM, Moza M, Ristimaki A, Soini Y, et al. Nrf2/Keap1 Pathway and Expression of Oxidative Stress Lesions 8-hydroxy-2'-deoxyguanosine and Nitrotyrosine in Melanoma. Anticancer Res. 2016;36(4):1497-506.

45. Robert C, Schachter J, Long GV, Arance A, Grob JJ, Mortier L, et al. Pembrolizumab versus Ipilimumab in Advanced Melanoma. N Engl J Med. 2015;372(26):2521-32.

46. Postow MA, Callahan MK, Wolchok JD. Immune Checkpoint Blockade in Cancer Therapy. J Clin Oncol. 2015;33(17):1974-82.

47. Cao J, Wan L, Hacker E, Dai X, Lenna S, Jimenez-Cervantes C, et al. MC1R is a potent regulator of PTEN after UV exposure in melanocytes. Mol Cell. 2013;51(4):409-22.

48. Zhu B, Zhang M, Williams EM, Keller C, Mansoor A, Davie JK. TBX2 represses PTEN in rhabdomyosarcoma and skeletal muscle. Oncogene. 2016;35(32):4212-24. 


\section{Figure 1}

PD-L1 is dramatically up-regulated by UVB exposure. (a) Human subjects were diagnosed with psoriasis vulgaris. Two lesions with the same level of lesional degree were selected for analysis in each subject. NB-UVB treatment $\left(300 \mathrm{~J} / \mathrm{m}^{2}\right)$ was provided to one lesion, with the alternate lesion sham treated. Skin biopsies were collected 6 hours after NB-UVB treatment. Tissue sections from the paired patient biopsies were then analyzed by IHC for PD-L1 ${ }^{+}$cells. (b, c) Histogram plots showing increased protein levels of PD-L1 with flow cytometry assay on b, human primary keratinocytes (HPKs) and c, human primary melanocytes (HPMs) after indicated time of UVB treatment $\left(100 \mathrm{~J} / \mathrm{m}^{2}\right)$. UVB-responsive phosphorylation of CHK1 and p38 were assayed with immunoblotting in (b), HPKs and (c), HPMs after indicated time of UVB treatment $\left(100 \mathrm{~J} / \mathrm{m}^{2}\right)$. (d, e) The upregulation of PD-L1 mRNA level was assayed with qRT-PCR in (d), HPKs and (e), HPMs after indicated time of UVB treatment $\left(100 \mathrm{~J} / \mathrm{m}^{2}\right)$. Data shown correspond to one representative experiment out of three independent experiments. $* * * p<0.001$. 


\section{Figure 2}

NRF2 is required for UVB-induced PD-L1 upregulation. (a) Schematic representation of the human PD-L1 locus, indicating the location of a NRF2-binding consensus element (NBE) of various mammalian species. The nucleotide positions are relative to the transcription initiation start site. (b) NRF2 upregulation was assayed by immunoblotting with NRF2 antibody in HPKs and HPMs at indicated time point after UVB exposure $\left(100 \mathrm{~J} / \mathrm{m}^{2}\right)$, with $\beta$-actin used as the loading control. (c) Linear correlation graph depicting the association between expression of PD-L1 and NRF2 based on IHC staining of human melanoma tissue array with $p<0.0001$, $\mathrm{r}=0.7998, \mathrm{n}=92$. (d-f) $\mathrm{Nrf}^{-/-}$mice were exposed to UVB with dose of $500 \mathrm{~J} / \mathrm{m}^{2}$ on dorsal skin. Total protein was isolated from the radiated skin to perform western blot assay with specific anti-PD-L1 antibodies (d), to detect PD-L1 transcript level with qRT-PCR (d), and protein level with flow cytometry assay using isolated dorsal skin cells (f). Three independent experiments were performed $* * p<0.01$. 


\section{Figure 3}

PD-L1 is a direct transcriptional target of NRF2. (a) NRF2 overexpression was confirmed by immunoblotting with NRF2 antibody in NRF2-overexpressing HPKs and HPMs. $\beta$-actin was used as the loading control. (b) The upregulated PD-L1 mRNA level was assayed by qRT-PCR in HPKs and HPMs transfected with empty vector (EV) or NRF2 expression vector (NRF2). (c), Histogram plots showing increased protein levels of PD-L1 with flow cytometry assay of HPKs and HPMs overexpressing EV or NRF2. Red line represents PD-L1 protein level of cells with empty vector, black line as for cells overexpressing NRF2, and gray line as for isotype controls. (d) NRF2 knockdown was confirmed by immunoblotting with specific NRF2 antibody in HPKs and HPMs with stable shNRF2. $\beta$-actin was used as the loading control. (e) PD-L1 mRNA upregulation was assayed by qRT-PCR in UVB-radiated HPKs and HPMs with or without shNRF2. (f) Histogram plots show PD-L1 expression in UVB-radiated HPKs and HPMs with shNRF2 by flow cytometry. (g) The binding of NRF2 protein to PD-L1 gene regulatory region was assayed by chromatin immunoprecipitation assay in HPMs 24 hours after UVB $\left(100 \mathrm{~J} / \mathrm{m}^{2}\right.$ ) exposure. NQO1 served as a known NRF2 transcriptional target (positive control) and $\beta$-actin served as a negative control. All values were normalized against the relative control IgG sample. (h) NRF2-activated PD-L1 transcription was measured by luciferase assay with PD-L1 reporters in the HKE293T cells. Constructs included basic pGL3 vector, pGL3 vector with PD-L1 promoter $(-281 /+43)$ upstream of luciferase gene, pGL3 vector with the promoter and wild-type enhancer $(+1845 /+1988)$ including NRF2 binding site $(+1932 /+1948)$ cloned downstream of luciferase gene, and pGL3 vector with the promoter and mutant enhancer $(+1845 /+1988)$ without NRF2 binding site. All values were normalized against pGL3 basic vector sample. Three independent experiments were performed. ${ }^{*} p<0.05$; $* * p<0.01 ; * * * p<0.001$. 


\section{Figure 4}

NRF2 levels in melanoma cells correlate with the anti-tumor reactivity of lymphocytes. (a) Target cells of B16 with NRF2 overexpression and LLC (control) cells were labeled with high and low concentration of CFSE, respectively. All these resulted cells were co-cultured with activated Pmel-1 T cells for 5 hours. Histogram plots show cytotoxicity of Pmel- 1 T cells induced by target melanoma cells. Quantifications were demonstrated. (b) Target B16 with NRF2 overexpression were co-cultured with activated Pmel-1 T cells. IFN- $\gamma$ in the culture supernatant was quantified with ELISA. (c) The transcript levels of IFN- $\gamma$, IL-2 and TNF $\alpha$ in activated Pmel-1 were assayed with RT-qPCR after co-culture. (d) B16 cells with shNRF2 were used as the target cells. The cytotoxicity assay in Pmel-1 was performed as in (a). (e) IFN- $\gamma$ in the culture supernatant was quantified with ELISA after co-culturing target B16 with stable shNRF2 with the activated Pmel-1 T cells. (f) RT-qPCR assay was performed to detect the transcript levels of IFN- $\gamma$, IL-2 and TNF $\alpha$ in activated Pmel-1 T cells co-cultured with B16 cells with stable shNRF2. Three independent experiments were performed. ${ }^{* *} p<0.01$ and $* * * p<0.001$. 


\section{Figure 5}

NRF2 depletion cooperates with PD-L1 blockade to treat melanoma. B16-BL6 cells $\left(2 \times 10^{4}\right.$ in $100 \mu \mathrm{l} \mathrm{PBS}$ ) with stable NRF2 silencing or control shRNA were s.c. injected into C57BL/6 mice ( $\mathrm{n}=5$ mice per group). IgG or PD- 1 antibody ( $5 \mathrm{mg} / \mathrm{kg}$ per animal) were i.p. administrated from day 10 to day 15. (a-b) For each group, the tumor growth curve (a) and tumor weight (b) were shown. (c) Flow cytometry assay was performed to detect the PD-L1 expression on tumor cells after allograft assay. (d-f) Population of $\mathrm{CD}^{+}\left(\mathrm{CD} 45^{+} \mathrm{CD}^{+} \mathrm{CD}^{+}\right.$) (d), $\mathrm{CD}^{+}\left(\mathrm{CD} 45^{+} \mathrm{CD}^{+} \mathrm{CD}^{+}\right)(\mathbf{e})$ and regulatory $\left(\mathrm{CD} 4^{+} \mathrm{CD} 25^{+} \mathrm{Foxp}^{+}\right) \mathrm{T}$ cells (f) were shown as a percentage of total infiltrating immune cells in the tumors. (g-h) Population of IFN $\gamma^{+} \mathrm{CD}^{+} \mathrm{T}(\mathbf{g})$ and granzyme $\mathrm{B}^{+}\left(\mathrm{GzmB}^{+}\right) \mathrm{CD}^{+}(\mathbf{h})$ cells of total tumor-infiltrating $\mathrm{CD}^{+}$ T cells were shown with flow cytometry assay. (i-j) Population of MDSC $\left(\mathrm{Gr} 1^{+} \mathrm{CD} 11 \mathrm{~b}^{+}\right)(\mathbf{i})$ and macrophage $\left(\mathrm{CD} 11 \mathrm{~b}^{+} \mathrm{F} 4 / 80^{+}\right)(\mathbf{j})$ in tumors were assayed, as well as the ratio of M1/M2 (j) in TILs. ${ }^{*} p<0.05 ; * * p<0.01 ; * * * p<0.001$. 